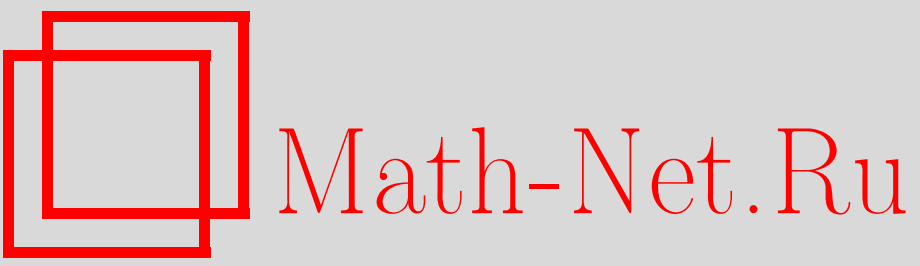

M. López-García, Characterization of solutions to the log-normal moment problem, Теория вероятн. и ее примен., 2010, том 55, выпуск 2, 387-391

DOI: https://doi.org/10.4213/tvp4211

Использование Общероссийского математического портала Math-Net.Ru подразумевает, что вы прочитали и согласны с пользовательским соглашением

http://www.mathnet.ru/rus/agreement

Параметры загрузки:

IP: 3.91 .87 .62

26 апреля 2023 г., 14:24:56 
(C) 2010 г.

\author{
LÓPEZ-GARCÍA M.*
}

\title{
CHARACTERIZATION OF SOLUTIONS TO THE LOG-NORMAL MOMENT PROBLEM
}

Хорошо известно, что интегрируемая функция на $(0, \infty)$, удовлетворяющая функциональному уравнению $f(q x)=q^{-1 / 2} x f(x), 0<q<1$, и такая, что $\int f=1$, является решением логнормальной проблемы моментов. В настоящей работе вводятся и характеризуются некоторые семейства решений логнормальной проблемы моментов, не удовлетворяющие упомянутому функциональному уравнению.

Ключевые слова и фразы: неопределенная проблема моментов, логнормальное распределение, гауссовское ядро.

1. Introduction. For a real-valued, measurable function $f$ defined on $(0, \infty)$, its $n$-th moment is defined as $s_{n}(f)=\int_{0}^{\infty} x^{n} f(x) d x, n \in \mathbf{Z}$. If $f$ is a real-valued, measurable function defined on $(0, \infty)$ with moment sequence $\left(q^{-n^{2} / 2}\right)_{n \in \mathbf{Z}}, 0<q<1$, then we say that $f$ is a solution to the log-normal moment problem.

If $f$ is a complex-valued, measurable function defined on $(0, \infty)$ such that

$$
\int_{0}^{\infty} x^{n} f(x) d x=q^{-n^{2} / 2} \quad \forall n \in \mathbf{Z}
$$

then $\operatorname{Re} f$ is a solution to the log-normal moment problem.

Throughout this paper we assume that $q \in(0,1)$ has been fixed, so we can write $q=e^{-\sigma^{2}}$ for some $\sigma>0$ fixed, and we will use that $\log _{q} x=\ln x / \ln q$. by

In [7] Stieltjes showed that the log-normal distribution with density on $(0, \infty)$ given

together with the densities $(a \in[-1,1])$

$$
d_{\sigma}(x)=\left(2 \pi \sigma^{2}\right)^{-1 / 2} x^{-1} \exp \left(-2^{-1} \sigma^{-2}(\ln x)^{2}\right)
$$

$$
d_{\sigma}(x)\left(1+a \sin \left(2 \pi \sigma^{-2} \ln x\right)\right) \geqslant 0,
$$

have all the moment sequence $\left(q^{-n^{2} / 2}\right)_{n \in \mathbf{Z}}$. Because the log-normal moment problem has no unique solution it is called M-indeterminate.

We recall that the so-called theta function is given by

$$
\theta(x, t)=(4 \pi t)^{-1 / 2} \sum_{n \in \mathbf{Z}} e^{-(x+n)^{2} /(4 t)} \quad \forall(x, t) \in \mathbf{R}_{+}^{2},
$$

see $[1$, p. 59].

When $\beta=0$ the following result is a consequence of observations made in [2]-[4], and characterizes a class of solutions to the log-normal moment problem.

Theorem 1. Let $\beta \in \mathbf{R}, f \in L^{1}(0, \infty)$. Then $f$ satisfies the functional equation

$$
f(q x)=q^{\beta-1 / 2} x f(x), \quad x>0,
$$

and $\int_{0}^{\infty} f(x) d x=q^{-\beta^{2} / 2}$ if and only if there exists a 1-periodic function $\varphi \in L^{1}(0,1)$ such that $f(x)=x^{\beta} d_{\sigma}(x)\left\{1+\varphi\left(\beta+\log _{q} x\right)\right\}$ and

$$
\int_{0}^{1} \varphi(x) \theta\left(x, 2^{-1} \sigma^{-2}\right) d x=0 .
$$

In any case, $s_{n}(f)=q^{-(n+\beta)^{2} / 2}$ for all $n \in \mathbf{Z}$.

* Instituto de Matemáticas, Universidad Nacional Autónoma de México, México, D.F., C.P. 04510, México; e-mail: flopez@matem.unam.mx 
When $\beta=0$ and $\varphi(x)=-a \sin (2 \pi x)$ we have the Stieltjes example. Example 2 given in Section 3 shows that $\varphi$ satisfies (2).

On the other hand, by setting $\beta=0$ and

$$
\varphi_{c}(x)=-1+q^{c^{2} / 2} \sigma^{2} M_{c}^{-1} \theta\left(-x+c, 2^{-1} \sigma^{-2}\right)^{-1},
$$

$c \in[0,1)$, we obtain the well-known solution (see [5, equality (1.9)])

$$
f_{c}(x)=\frac{x^{c-1}}{M_{c}(q ; q)_{\infty}\left(-q^{-c+1 / 2} x ; q\right)_{\infty}\left(-q^{c+1 / 2} x^{-1} ; q\right)_{\infty}}
$$

to the log-normal moment problem, where $M_{c}$ is the constant that makes $\int_{0}^{\infty} f_{c}(x) d x=1$, and $(p ; q)_{\infty}=\prod_{n=0}^{\infty}\left(1-p q^{n}\right)$. To get more examples see [5].

$\mathrm{R}$ e $\mathrm{m}$ a $\mathrm{rk} 1$. If $\varphi$ satisfies (2), then so does $a \varphi, a \in \mathbf{C}$. Moreover, if $\varphi$ is realvalued and bounded below (above), then there is $a \in \mathbf{R}$ such that $1+a \varphi \geqslant 0$. Hence in this case a probability density function $f$ can be obtained by a standard normalizing procedure.

Next, we introduce the fundamental solution of the heat equation on $\mathbf{R}_{+}^{2}$, the so-called Gauss kernel $K(x, t)=(4 \pi t)^{-1 / 2} e^{-x^{2} /(4 t)}>0$.

When $\beta=0$ the following results provide another class of solutions to the log-normal moment problem but they do not satisfy the functional equation (1). We denote by $\Phi$ the Fourier transform operator.

Theorem 2. Let $\beta \in \mathbf{R}$, and $F \in L^{2}(\mathbf{R})$. The function $g(x)=x^{\beta} d_{\sigma}(x)\{1+F(\beta+$ $\left.\left.\log _{q} x\right)\right\}$ satisfies $s_{n}(g)=q^{-(n+\beta)^{2} / 2}$ for all $n \in \mathbf{Z}$ if and only if

$$
\sum_{n \in \mathbf{Z}} K\left(\xi+2 n \pi, 2^{-1} \sigma^{2}\right)(\Phi F)(\xi+2 n \pi)=0 \quad \text { for almost all } \xi \in \mathbf{R} .
$$

If $F$ is not the zero function, then $g$ does not satisfy the functional equation (1).

If $\gamma \in L^{2}(\mathbf{R})$ has compact support and satisfies

$$
\sum_{n \in \mathbf{Z}} \gamma(\xi+2 n \pi)=0 \quad \text { for almost all } \quad \xi \in \mathbf{R}
$$

then the function $F \in L^{2}(\mathbf{R})$ given by $\Phi F=\gamma / K\left(\cdot, 2^{-1} \sigma^{2}\right)$ satisfies (3). For instance, consider $\phi \in C^{\infty}(\mathbf{R})$ with $\operatorname{supp} \phi \subset[0,2 \pi]$, then $\gamma(\xi)=\phi(\xi)-\phi(\xi-2 \pi)$ fulfills (4), and the corresponding function $F$ is a rapidly decreasing function.

$\mathrm{R}$ e $\mathrm{m}$ a $\mathrm{rk} 2$. If $F$ satisfies (3), then so does $a F, a \in \mathbf{C}$. Moreover, if $F$ is realvalued and bounded below (above), then there is $a \in \mathbf{R}$ such that $1+a F \geqslant 0$. Hence a probability density function can be obtained.

Theorem 3. Let $\beta \in \mathbf{R}, \alpha \in \mathbf{R} \backslash 2 \pi \mathbf{Z}, \lambda \in \mathbf{C}$, and let $f$ be a 1-periodic function in $L^{1}(0,1)$. The function $g(x)=x^{\beta} d_{\sigma}(x)\left\{1+F\left(\beta+\log _{q} x\right)\right\}$, with $F(x)=(\lambda+f(x)) e^{i \alpha x}$, satisfies $s_{n}(g)=q^{-(n+\beta)^{2} / 2}$ for all $n \in \mathbf{Z}$ if and only if

$$
\sqrt{2 \pi \sigma^{2}} K\left(\alpha, 2^{-1} \sigma^{2}\right) \lambda=-\int_{\mathbf{R}} K\left(x, 2^{-1} \sigma^{-2}\right) f(x) e^{i \alpha x} d x .
$$

If $F$ is not the zero function, then $g$ does not satisfy the functional equation (1).

$\mathrm{R}$ e $\mathrm{m}$ a $\mathrm{rk} 3$. When Re $F$ is bounded below (above), with $\lambda$ as before, there exists $a \in \mathbf{R}$ such that $1+a \operatorname{Re} F \geqslant 0$, and a probability density function can be obtained from the corresponding function $\operatorname{Re} g$. result.

By setting $\beta=0, f(x)=\cos (2 \pi x)$ in the previous theorem we obtain the following 
Corollary 1. The function $d_{\sigma}(x)\left\{1+\left(\lambda+\cos \left(2 \pi \log _{q} x\right)\right) \cos \left(\alpha \log _{q} x\right)\right\}$ is a solution to the log-normal moment problem that does not satisfy the functional equation (1), provided that $\alpha \in \mathbf{R} \backslash 2 \pi \mathbf{Z}$ and

$$
\lambda=-e^{\sigma^{-2} \alpha^{2} / 2} \int_{\mathbf{R}} K\left(x, 2^{-1} \sigma^{-2}\right) \cos (2 \pi x) \cos (\alpha x) d x .
$$

Theorem 4. Let $\beta \in \mathbf{R}$. There exists no function $h \in L^{2}(\mathbf{R})$ with $g(x)=$ $x^{\beta} d_{\sigma}(x) h\left(\beta+\log _{q} x\right)$ satisfying $s_{n}(g)=q^{-(n+\beta)^{2} / 2}$ for all $n \in \mathbf{Z}$.

This note is organized as follows. Preliminaries are given in the following section, and the last section contains the proofs of the theorems.

2. Notation and preliminaries. We follow the notation in [6, Chap. 9]. For $f \in$ $L^{1}(\mathbf{R})$, we define its Fourier transform as

$$
(\Phi f)(\xi)=\int_{-\infty}^{\infty} f(x) e^{-i x \xi} \frac{d x}{\sqrt{2 \pi}} .
$$

The Fourier transform operator $\Phi$ is a bijective map from $L^{2}(\mathbf{R})$ onto $L^{2}(\mathbf{R})$.

If $f, g \in L^{2}(\mathbf{R})$, then the Parseval formula holds:

$$
\int_{-\infty}^{\infty} f(x) \overline{g(x)} \frac{d x}{\sqrt{2 \pi}}=\int_{-\infty}^{\infty}(\Phi f)(\xi) \overline{(\Phi g)(\xi)} \frac{d \xi}{\sqrt{2 \pi}} .
$$

The translation operators $\tau_{x}$ are defined by

$$
\left(\tau_{x} f\right)(y)=f(y-x),
$$

and for $f \in L^{1}(\mathbf{R})$ we have $\Phi\left(\tau_{x} f\right)(\xi)=e^{i x \xi}(\Phi f)(\xi)$.

For $(x, t) \in \mathbf{R}_{+}^{2}$, let (see $[1$, p. 33, 59])

$$
\begin{aligned}
K(x, t) & =(2 \pi)^{-1 / 2} \Phi^{-1}\left(e^{-t \xi^{2}}\right)(x)=(4 \pi t)^{-1 / 2} e^{-x^{2} /(4 t)}, \\
\theta(x, t) & =\sum_{n \in \mathbf{Z}} K(x+n, t)=\sum_{n \in \mathbf{Z}} e^{-4 \pi^{2} n^{2} t+2 \pi n i x} .
\end{aligned}
$$

The positive functions $K, \theta$ satisfy the heat equation on $\mathbf{R}_{+}^{2}$. Clearly $\theta(\cdot, t)$ is a 1 -periodic continuous function for all $t>0$. Moreover,

$$
\int_{0}^{1} \theta(x, t) d x=\int_{\mathbf{R}} K(x, t) d x=1 \quad \forall t>0 .
$$

When $\beta=1 / 2$ the following observation can be found in [3], [4].

Proposition 1. Let $0<q<1, \beta \in \mathbf{R}$ fixed. If $f \in L^{1}(0, \infty)$ satisfies the functional equation (1), then $s_{n}(f)=q^{-n^{2} / 2-\beta n} \int_{0}^{\infty} f(x) d x, n \in \mathbf{Z}$.

$\mathrm{P}$ r o o f. By induction we can see that $f$ satisfies the functional equation

$$
f\left(q^{n} x\right)=q^{n^{2} / 2+(\beta-1) n} x^{n} f(x) \quad \forall n \in \mathbf{Z},
$$

thus

$$
\int_{0}^{\infty} x^{n} f(x) d x=q^{-n^{2} / 2-\beta n} \int_{0}^{\infty} q^{n} f\left(q^{n} x\right) d x=q^{-n^{2} / 2-\beta n} \int_{0}^{\infty} f(x) d x .
$$

$\mathrm{E} \times \mathrm{m} \mathrm{a} \mathrm{l}$ e 1 . For $\beta \in \mathbf{R}$, the positive function $x^{\beta} d_{\sigma}(x)$ is integrable on $(0, \infty)$ and satisfies the functional equation (1).

Proposition 2. Let $\beta \in \mathbf{R}, h \in L^{1}\left(\mathbf{R}, q^{(x-n)^{2} / 2} d x\right)$ for all $n \in \mathbf{Z}$. Then the function $g(x)=x^{\beta} d_{\sigma}(x) h\left(\beta+\log _{q} x\right)$ satisfies $s_{n}(g)=q^{-(n+\beta)^{2} / 2}$ for all $n \in \mathbf{Z}$ if and only if

$$
\int_{\mathbf{R}} K\left(x, 2^{-1} \sigma^{-2}\right) h(x+n) d x=1 \quad \forall n \in \mathbf{Z}
$$


P r o o f. Since $q=e^{-\sigma^{2}}$, we can write $d_{\sigma}(x)=\left(2 \pi \sigma^{2}\right)^{-1 / 2} x^{-1} q^{\left(\log _{q} x\right)^{2} / 2}$. Notice that $x^{\lambda} d_{\sigma}(x)=q^{\lambda-\lambda^{2} / 2} d_{\sigma}\left(x q^{\lambda}\right)$ for all $x>0, \lambda \in \mathbf{R}$, so by making the change of variable $y=n+\beta+\log _{q} x$, we obtain

$$
\begin{aligned}
s_{n}(g) & =q^{-(\beta+n)^{2} / 2} \int_{-\infty}^{\infty}\left(2 \pi \sigma^{-2}\right)^{-1 / 2} q^{y^{2} / 2} h(y-n) d y \\
& =q^{-(\beta+n)^{2} / 2} \int_{-\infty}^{\infty} K\left(y, 2^{-1} \sigma^{-2}\right) h(y-n) d y,
\end{aligned}
$$

and the result follows.

$\mathrm{R}$ e $\mathrm{m}$ a $\mathrm{rk}$ 4. In $q$-calculus a function $f$ defined on $(0, \infty)$ is $q$-periodic if $f(q x)=$ $f(x)$ for all $x>0$. If $f$ is a $q$-periodic function defined on $(0, \infty)$, then $\psi(x)=f\left(q^{x}\right)$ is a 1 -periodic function (in the usual sense) on $\mathbf{R}$. So, we have that $f$ is a $q$-periodic function if and only if there exists a 1-periodic function $\psi$ such that $f(x)=\psi\left(\log _{q} x\right)$.

$\mathrm{R}$ e $\mathrm{m}$ a $\mathrm{rk} 5$. If $f, g:(0, \infty) \rightarrow \mathbf{R}$ satisfy the functional equation (1) and $g$ is a positive function, then $(f / g)(x)=(f / g)(q x)$, therefore $f / g$ is a $q$-periodic function. By the previous remark there exists a 1-periodic function $\psi$ such that $(f / g)(x)=\psi\left(\log _{q} x\right)$. In particular, we can write $f(x)=x^{\beta} d_{\sigma}(x) \psi\left(\log _{q} x\right)$, where $\psi$ is a 1-periodic function.

3. Proofs of Theorems 1-4.

$\mathrm{P}$ r o of of $\mathrm{T} \mathrm{h}$ e o r e $\mathrm{m} 1$. Remark 5 implies that there exists a 1-periodic function $\psi$ such that $f(x)=x^{\beta} d_{\sigma}(x) \psi\left(\log _{q} x\right), x>0$. By (9), with $h=\psi(\cdot-\beta)$, we obtain

$$
\begin{aligned}
q^{-\beta^{2} / 2} & =s_{0}(f)=q^{-\beta^{2} / 2} \int_{\mathbf{R}} K\left(x, 2^{-1} \sigma^{-2}\right) \psi(x-\beta) d x \\
& =q^{-\beta^{2} / 2} \int_{0}^{1} \psi(x-\beta) \theta\left(x, 2^{-1} \sigma^{-2}\right) d x .
\end{aligned}
$$

By (7) we have that $\varphi(x)=-1+\psi(x-\beta)$ is a 1-periodic function satisfying (2).

On the other hand, if $\varphi$ is a 1-periodic function, then $f(x)=x^{\beta} d_{\sigma}(x)\left\{1+\varphi\left(\beta+\log _{q} x\right)\right\}$ satisfies (1). The previous computations, (2), and (7) show that $\int_{0}^{\infty} f(x) d x=q^{-\beta^{2} / 2}$. Theorem 1 is proved.

if

Ex a m p l e 2. If $\varphi(x)=\sum_{n \in \mathbf{Z}} c_{n} e^{2 \pi n i x} \in L^{2}(\mathbf{T})$, then $\varphi$ satisfies (2) if and only

$$
\sum_{n \in \mathbf{Z}} c_{n} e^{-2 \sigma^{-2} \pi^{2} n^{2}}=0
$$

(see (6)). When $c_{-1}=-a i / 2, c_{0}=0, c_{1}=a i / 2, c_{n}=0$ for all $|n|>1, a \in[-1,1]$, we have the Stieltjes example with $\varphi(x)=-a \sin (2 \pi x)$.

$\mathrm{Pr}$ o of of $\mathrm{Th}$ e o r e $\mathrm{m}$ 2. Since $q^{(x-n)^{2} / 2}$ is a rapidly decreasing function, we have $q^{(x-n)^{2} / 2} F \in L^{1}(\mathbf{R})$ for all $n \in \mathbf{Z}$. The Parseval formula and (5) imply that

$$
\begin{aligned}
a_{n} & :=\int_{\mathbf{R}} K\left(x, 2^{-1} \sigma^{-2}\right) F(x+n) d x \\
& =\int_{\mathbf{R}} \Phi^{-1}\left(e^{-2^{-1} \sigma^{-2} \xi^{2}}\right)(x)\left(\tau_{-n} F\right)(x) \frac{d x}{\sqrt{2 \pi}} \\
& =\int_{\mathbf{R}} e^{-2^{-1} \sigma^{-2} \xi^{2}}(\Phi F)(\xi) e^{-i n \xi} \frac{d \xi}{\sqrt{2 \pi}} \\
& =\int_{0}^{2 \pi}\left\{\sum_{m \in \mathbf{Z}} e^{-2^{-1} \sigma^{-2}(\xi+2 \pi m)^{2}}(\Phi F)(\xi+2 \pi m)\right\} e^{-i n \xi} \frac{d \xi}{\sqrt{2 \pi}} .
\end{aligned}
$$

Therefore $a_{n}$ is a multiple of the $n$-th Fourier coefficient of the function $\sum_{m \in \mathbf{Z}} K(\xi+$ $\left.2 \pi m, 2^{-1} \sigma^{2}\right)(\Phi F)(\xi+2 \pi m) \in L^{1}(\mathbf{T})$. By using Proposition 2, with $h=1+F$, and (7) we get that $a_{n}=0$ for all $n \in \mathbf{Z}$ and the result follows. 
If $g$ satisfies the functional equation (1), Remark 5 implies that $F$ is a 1-periodic function in $L^{2}(\mathbf{R})$, therefore $F=0$ a.e. in $\mathbf{R}$. Theorem 2 is proved.

P r o of of $\mathrm{Th}$ e or e m 3. Clearly $q^{(x-n)^{2} / 2} F \in L^{1}(\mathbf{R})$ for all $n \in \mathbf{Z}$, since

$$
\int_{\mathbf{R}} K\left(x-n, 2^{-1} \sigma^{-2}\right)|f(x)| d x=\int_{0}^{1} \theta\left(x, 2^{-1} \sigma^{-2}\right)|f(x)| d x<\infty \quad \forall n \in \mathbf{Z} .
$$

Proposition 2 and (7) imply that

$$
\lambda \int_{\mathbf{R}} K\left(x, 2^{-1} \sigma^{-2}\right) e^{i \alpha x} d x=\int_{\mathbf{R}} K\left(x, 2^{-1} \sigma^{-2}\right) f(x) e^{i \alpha x} d x .
$$
get that

By (5), noting that $\Phi^{-1}\left(e^{-t \xi^{2}}\right)(x)=\Phi\left(e^{-t \xi^{2}}\right)(x)$ and using the inversion formula we

$$
\begin{aligned}
\int_{\mathbf{R}} K\left(x, 2^{-1} \sigma^{-2}\right) e^{i \alpha x} d x & =\int_{\mathbf{R}} \Phi\left(e^{-2^{-1} \sigma^{-2} \xi^{2}}\right)(x) e^{i \alpha x} \frac{d x}{\sqrt{2 \pi}} \\
& =\left.\Phi^{-1} \Phi\left(e^{-2^{-1} \sigma^{-2} \xi^{2}}\right)\right|_{\xi=\alpha}=e^{-2^{-1} \sigma^{-2} \alpha^{2}}
\end{aligned}
$$

If $F$ is not the zero function, then it is not a 1-periodic function, and Remark 5 implies that $g$ does not satisfy the functional equation (1). Theorem 3 is proved.

P r o of of $\mathrm{Th}$ e o r e m 4. Let $h \in L^{2}(\mathbf{R})$, then $q^{(x-n)^{2} / 2} h \in L^{1}(\mathbf{R})$ for all $n \in \mathbf{Z}$. By the Parseval formula we have that $a_{n}=\int_{\mathbf{R}} K\left(x, 2^{-1} \sigma^{-2}\right) h(x+n) d x$ is a multiple of the $n$-th Fourier coefficient of the function $\sum_{m \in \mathbf{Z}} K\left(\xi+2 \pi m, 2^{-1} \sigma^{2}\right)(\Phi h)(\xi+$ $2 \pi m) \in L^{1}(\mathbf{T})$. Proposition 2 would imply that $a_{n}=1$ for all $n \in \mathbf{Z}$, but this contradicts the Riemann-Lebesgue lemma. Theorem 4 is proved.

Acknowledgment. I thank the anonymous referee for carefully reading the original manuscript and for their suggestions.

\section{СПИСОК ЛИТЕРАТУРЫ}

1. Cannon J. R. The One-Dimensional Heat Equation. Reading: Addison-Wesley, 1984, $483 \mathrm{p}$.

2. Chihara T.S. On generalized Stieltjes-Wigert and related orthogonal polynomials. J. Comput. Appl. Math., 1979, v. 5, № 4, p. 291-297.

3. Chihara T.S. A characterization and a class of distribution functions for the StieltjesWigert polynomials. - Canad. Math. Bull., 1970, v. 13, p. 529-532.

4. Christiansen J.S. The moment problem associated with the Stieltjes-Wigert polynomials. - J. Math. Anal. Appl., 2003, v. 277, № 1, p. 218-245.

5. Gómez R., López-García M. A family of heat functions as solutions of indeterminate moment problems. - Int. J. Math. Math. Sci., 2007, v. 2007, Article ID 41526, 11 p.

6. Rudin W. Real and Complex Analysis. New York: McGraw-Hill series in higher mathematics, 1987, $416 \mathrm{p}$.

7. Stieltjes T. J. Recherches sur les fractions continues. - Ann. Fac. Sci. Toulouse, 1894, v. 8 , p. $1-122$; 1895 , v. 9 , p. 5-47; рус. пер.: Стилтьес Т.И. Исследования о непрерывных дробях. Киев-Харьков: ОНТИ, 1936, 155 с.

Поступила в редакцию

10.XI.2007

Исправленный вариант

03.VIII. 2008 